Research Article

\title{
Convergence Results for the Gaussian Mixture Implementation of the Extended-Target PHD Filter and Its Extended Kalman Filtering Approximation
}

\author{
Feng Lian, Chongzhao Han, Jing Liu, and Hui Chen \\ Ministry of Education Key Laboratory for Intelligent Networks and Network Security (MOE KLINNS), \\ School of Electronics and Information Engineering, Xi'an Jiaotong University, Xi'an 710049, China \\ Correspondence should be addressed to Feng Lian, lianfeng1981@gmail.com
}

Received 23 February 2012; Accepted 31 May 2012

Academic Editor: Qiang Ling

Copyright (C) 2012 Feng Lian et al. This is an open access article distributed under the Creative Commons Attribution License, which permits unrestricted use, distribution, and reproduction in any medium, provided the original work is properly cited.

\begin{abstract}
The convergence of the Gaussian mixture extended-target probability hypothesis density (GMEPHD) filter and its extended Kalman (EK) filtering approximation in mildly nonlinear condition, namely, the EK-GM-EPHD filter, is studied here. This paper proves that both the GM-EPHD filter and the EK-GM-EPHD filter converge uniformly to the true EPHD filter. The significance of this paper is in theory to present the convergence results of the GM-EPHD and EK-GM-EPHD filters and the conditions under which the two filters satisfy uniform convergence.
\end{abstract}

\section{Introduction}

The problem of extended-target tracking (ETT) [1,2] arises because of the sensor resolution capacities [3], the high density of targets, the sensor-to-target geometry, and so forth. For targets in near field of a high-resolution sensor, the sensor is able to receive more than one measurement (observation, or detection) at each time from different corner reflectors of a single target. In this case, the target is no longer known as a point object, which at most causes one detection at each time. It is called extended target. ETT is very valuable for many real applications $[4,5]$, such as ground or littoral surveillance, robotics, and autonomous weapons.

The ETT problem has attracted great interest in recent years. Some approaches [6, 7] have been proposed for tracking a known and fixed number of the extended targets without clutter. Nevertheless, for the problem of tracking an unknown and varying number of the extended targets in clutter, most of the association-based approaches [8], such as nearest neighbor, joint probabilistic data association, and multiple hypothesis tracking, would no longer be applicable straightforwardly owing to their underlying assumption of point objects. 
Recently, the random-finite-set- (RFS-) based tracking approaches [9] have attracted extensive attention because of their lots of merits. The probability hypothesis density (PHD) [10] filter, developed by Mahler for tracking multiple point targets in clutter, has been shown to be computationally tractable alternative to full multitarget filter in the RFS framework. The sequential Monte Carlo (SMC) implementation for the PHD filter was devised by Vo et al. [11]. Then, Vo and Ma [12] devised the Gaussian mixture (GM) implementation for the PHD filter under the linear, Gaussian assumption on target dynamics, birth process, and sensor model. Actually the original intention of the PHD filter devised by Clark and Godsill is to address nonconventional tracking problems, that is, tracking in high target density, tracking closely spaced targets, and detecting targets of interest in a dense clutter background [13]. So it is especially suitable for the ETT problem.

Given the Poisson likelihood model for the extended target [14], Mahler developed the theoretically rigorous PHD filter for the ETT problem in 2009 [15]. Under the linear, Gaussian assumption, the GM implementation for the extended-target PHD (EPHD) filter was proposed by Granström et al. [16], in 2010. Similar to the point-target GM-PHD filter, the GMEPHD filter can also be extended to accommodate mildly nonlinear target dynamics using the extended Kalman (EK) filtering [17] approximation. The extension is called EK-GM-EPHD filter. Experimental results showed the EK-GM-EPHD filter was capable of tracking multiple humans, each of which gave rise to, on average, 10 measurements at each scan and was therefore treated as an extended target, using a SICK LMS laser range sensor [16].

Although the GM-EPHD and EK-GM-EPHD filters have been successfully used for many real-world problems, there have been no results showing the convergence for the two filters. The convergence results on point-target particle-PHD and GM-PHD filters [18, 19] do not apply directly for the GM-EPHD and EK-GM-EPHD filters because of the significant difference between the measurement update steps of the PHD and EPHD filters. Therefore, to ensure the more successful and extensive applications of the EPHD filter to "real-life" problems, it is necessary to answer the following question: do the GM-EPHD and EK-GMEPHD filters converge asymptotically toward the optimal filter and in what sense?

The answer can actually be derived from Propositions 3.2 and 3.3 in this paper. Proposition 3.2 demonstrates the uniform convergence [20-22] of the errors for the measurement update step of the GM-EPHD filter. In other words, given simple sufficient conditions, the approximation error of the measurement-updated EPHD by a sum of Gaussians is proved to converge to zero as the number of Gaussians in the mixture tends to infinity. In addition, the uniform convergence results for the measurement update step of the EK-GM-EPHD filter are derived from Proposition 3.3.

\section{EPHD and GM-EPHD Filters}

At time $k$, let $\mathbf{x}_{k}$ be the state vector of a single extended target, and $\mathbf{z}_{k}$ a single measurement vector received by sensor. Multiple extended-target states and sensor measurements can, respectively, be represented as finite sets $X_{k}=\left\{\mathbf{x}_{i, k}\right\}_{i=1}^{n_{k}}$ and $Z_{k}=\left\{\mathbf{z}_{i, k}\right\}_{i=1}^{m_{k}}$, where $n_{k}$ and $m_{k}$ denote the number of the extended targets and sensor measurements, respectively. A Poisson model is used to describe the likelihood function for the extended target according to Gilholm et al. [14]:

$$
l_{Z_{k}}\left(\mathbf{x}_{k}\right)=e^{-\gamma\left(\mathbf{x}_{k}\right)} \prod_{\mathbf{z}_{k} \in Z_{k}} \gamma\left(\mathbf{x}_{k}\right) \phi_{\mathbf{z}_{k}}\left(\mathbf{x}_{k}\right),
$$


where $\phi_{\mathbf{z}_{k}}\left(\mathbf{x}_{k}\right)$ denotes the single-measurement single-target likelihood density; $\gamma\left(\mathbf{x}_{k}\right)$ denotes the expected number of measurements arising from an extended target.

The clutter is modeled as a Poisson RFS with the intensity $\kappa_{k}\left(\mathbf{z}_{k}\right)=\lambda_{k} c_{k}\left(\mathbf{z}_{k}\right)$, where $\lambda_{k}$ is the average clutter number per scan and $c_{k}\left(\mathbf{z}_{k}\right)$ is the density of clutter spatial distribution.

Given the Poisson likelihood model for the extended targets, Mahler derived the EPHD filter using finite-set statistics [15, 23]. The prediction equations of the EPHD filter are identical to those of the point-target PHD filter [10]. The EPHD measurement update equations are

$$
\begin{aligned}
& v_{k}\left(\mathbf{x}_{k}\right)=\left(1-p_{D, k}\left(\mathbf{x}_{k}\right)+e^{-\gamma\left(\mathbf{x}_{k}\right)} p_{D, k}\left(\mathbf{x}_{k}\right)+\sum_{\oint_{k} \angle Z_{k}} \omega_{\wp_{k}} \sum_{W_{k} \in \mathfrak{\wp}_{k}} \frac{\eta_{W_{k}}\left(\mathbf{x}_{k}\right)}{d_{W_{k}}}\right) v_{k \mid k-1}\left(\mathbf{x}_{k}\right), \\
& \eta_{W_{k}}\left(\mathbf{x}_{k}\right)=p_{D, k}\left(\mathbf{x}_{k}\right) e^{-\gamma\left(\mathbf{x}_{k}\right)} \prod_{\mathbf{z}_{k} \in W_{k}} \frac{\gamma\left(\mathbf{x}_{k}\right) \phi_{\mathbf{z}_{k}}\left(\mathbf{x}_{k}\right)}{\lambda_{k} c_{k}\left(\mathbf{z}_{k}\right)}, \\
& \omega_{\mathfrak{\wp}_{k}}=\frac{\prod_{W_{k} \in \mathfrak{\phi}_{k}} d_{W_{k}}}{\sum_{\mathfrak{\wp}_{k}^{\prime} \angle Z_{k}} \prod_{W_{k}^{\prime} \in \mathcal{E}_{k}^{\prime}} d_{W_{k}^{\prime}}} ; \quad d_{W_{k}}=\delta_{\left|W_{k}\right|, 1}+\left\langle v_{k \mid k-1}, \eta_{W_{k}}\right\rangle \text {, }
\end{aligned}
$$

where $p_{D, k}\left(\mathbf{x}_{k}\right)$ denotes the probability that the set of observations from the extended target will be detected at time $k ; \mathfrak{\wp}_{k} \angle Z_{k}$ denotes that $\wp_{k}$ partitions set $Z_{k}$ [15], for example, let $Z=\left\{\mathbf{z}_{1}, \mathbf{z}_{2}, \mathbf{z}_{3}\right\}$, then the partitions of $Z$ are $\wp_{1}=\left\{\left\{\mathbf{z}_{1}, \mathbf{z}_{2}, \mathbf{z}_{3}\right\}\right\}, \wp_{2}=\left\{\left\{\mathbf{z}_{1}\right\},\left\{\mathbf{z}_{2}\right\},\left\{\mathbf{z}_{3}\right\}\right\}, \wp_{3}=$ $\left\{\left\{\mathbf{z}_{1}, \mathbf{z}_{2}\right\},\left\{\mathbf{z}_{3}\right\}\right\}, \wp_{4}=\left\{\left\{\mathbf{z}_{1}, \mathbf{z}_{3}\right\},\left\{\mathbf{z}_{2}\right\}\right\}$, and $\wp_{5}=\left\{\left\{\mathbf{z}_{1}\right\},\left\{\mathbf{z}_{2}, \mathbf{z}_{3}\right\}\right\} ;\left|W_{k}\right|$ denotes the cardinality of the set $W_{k} ; \delta_{\left|W_{k}\right|, 1}=1$ if $\left|W_{k}\right|=1$, and $\delta_{\left|W_{k}\right|, 1}=0$ otherwise; the notation $\langle\cdot, \cdot\rangle$ is the usual inner product. The measure in $\langle\cdot, \cdot\rangle$ of $(2.4)$ is continuous, it defines the integral inner product

$$
\left\langle v_{k \mid k-1}, \eta_{W_{k}}\right\rangle=\int v_{k \mid k-1}\left(\mathbf{x}_{k}\right) \eta_{W_{k}}\left(\mathbf{x}_{k}\right) d \mathbf{x}_{k} .
$$

By making the same six assumptions that are made in [12] and the additional assumption that $\gamma\left(\mathbf{x}_{k}\right)$ can be approximated as functions of the mean of the individual Gaussian components, Granström et al. proposed the GM-EPHD filter [16]. At time $k$, let $v_{k \mid k-1}^{J_{k \mid k-1}}$ denote the GM approximation to the predicted EPHD $v_{k \mid k-1}$ with $J_{k \mid k-1}$ Gaussian components, and $v_{k}^{J_{k}}$ the GM approximation to the measurement-updated EPHD $v_{k}$ with $J_{k}$ Gaussian components. The prediction equations of the GM-EPHD filter are identical to those of point-target GM-PHD filter [12]. The GM-EPHD measurement update equations are as follows.

Let the predicted EPHD be a GM of the form

$$
v_{k \mid k-1}^{J_{k \mid k-1}}\left(\mathbf{x}_{k}\right)=\sum_{i=1}^{J_{k \mid k-1}} w_{k \mid k-1}^{(i)} \mathcal{N}\left(\mathbf{x}_{k} \mid \mathbf{m}_{k \mid k-1}^{(i)}, \mathbf{P}_{k \mid k-1}^{(i)}\right)
$$

where $\mathcal{N}(\cdot \mid \mathbf{m}, \mathbf{P})$ denotes the density of Gaussian distribution with the mean $\mathbf{m}$ and covariance $\mathbf{P}$.

Then, the measurement-updated EPHD is a GM given by 


$$
v_{k}^{J_{k}}\left(\mathbf{x}_{k}\right)=v_{k}^{N D, J_{k \mid k-1}}\left(\mathbf{x}_{k}\right)+\sum_{\wp_{k} \angle Z_{k} W_{k} \in \xi_{k}} v_{k}^{D J_{k \mid k-1}}\left(\mathbf{x}_{k}, W_{k}\right)
$$

where $v_{k}^{N D, J_{k \mid k-1}}\left(\mathbf{x}_{k}\right)$ denotes the Gaussian components handling no detections,

$$
\begin{gathered}
v_{k}^{N D, J k \mid k-1}\left(\mathbf{x}_{k}\right)=\sum_{j=1}^{J_{k \mid k-1}} w_{k}^{(j)} \mathcal{N}\left(\mathbf{x}_{k} \mid \mathbf{m}_{k \mid k-1}^{(j)}, \mathbf{P}_{k \mid k-1}^{(j)}\right) \\
w_{k}^{(j)}=\left(1-\left(1-e^{-\gamma\left(\mathbf{m}_{k \mid k-1}^{(j)}\right)}\right) p_{D, k}\right) w_{k \mid k-1^{\prime}}^{(j)} \\
\mathbf{m}_{k}^{(j)}=\mathbf{m}_{k \mid k-1}^{(j)} ; \quad \mathbf{P}_{k}^{(j)}=\mathbf{P}_{k \mid k-1^{\prime}}^{(j)}
\end{gathered}
$$

and $v_{k}^{D, J_{k \mid k-1}}\left(\mathbf{x}_{k}, W_{k}\right)$ denotes the Gaussian components handling detected targets

$$
\begin{aligned}
& v_{k}^{D, J_{k \mid k-1}}\left(\mathbf{x}_{k}, W_{k}\right)=\sum_{j=1}^{J_{k \mid k-1}} w_{k}^{(j)} \mathcal{N}\left(\mathbf{x}_{k} \mid \mathbf{m}_{k}^{(j)}, \mathbf{P}_{k}^{(j)}\right), \\
& w_{k}^{(j)}=w_{\wp_{k}}^{J_{k \mid k-1}} \frac{\eta_{W_{k}}\left(\mathbf{m}_{k \mid k-1}^{(j)}\right)}{d_{W_{k}}^{J_{k \mid k-1}}} w_{k \mid k-1}^{(j)} \\
& \omega_{\mathscr{\wp}_{k}}^{J_{k \mid k-1}}=\frac{\prod_{W_{k} \in \mathscr{\ell}_{k}} d_{W_{k}}^{J_{k \mid k-1}}}{\sum_{\mathscr{\ell}_{k}^{\prime} \angle Z_{k}} \prod_{W_{k}^{\prime} \in \mathcal{\wp}_{k}^{\prime}} d_{W_{k}^{\prime}}^{J_{k \mid k-1}}} ; \quad d_{W_{k}}^{J_{k \mid k-1}}=\delta_{\left|W_{k}\right|, 1}+\left\langle v_{k \mid k-1}^{J_{k \mid k-1}}, \eta_{W_{k}}\right\rangle, \\
& \eta_{W_{k}}\left(\mathbf{m}_{k \mid k-1}^{(j)}\right)=p_{D, k} e^{-\gamma\left(\mathbf{m}_{k \mid k-1}^{(j)}\right)} \prod_{\mathbf{z}_{k} \in W_{k}} \frac{\gamma\left(\mathbf{m}_{k \mid k-1}^{(j)}\right) \phi_{\mathbf{z}_{k}}\left(\mathbf{m}_{k \mid k-1}^{(j)}\right)}{\lambda_{k} c_{k}\left(\mathbf{z}_{k}\right)}, \\
& \phi_{\mathbf{z}_{k}}\left(\mathbf{m}_{k \mid k-1}^{(j)}\right)=\mathcal{N}\left(\mathbf{z}_{k} \mid \mathbf{H}_{k} \mathbf{m}_{k \mid k-1}^{(j)}, \mathbf{R}_{k}+\mathbf{H}_{k} \mathbf{P}_{k \mid k-1}^{(j)} \mathbf{H}_{k}^{T}\right), \\
& \mathbf{K}_{k}^{(j)}=\mathbf{P}_{k \mid k-1}^{(j)} \overline{\mathbf{H}}_{k}^{T}\left(\overline{\mathbf{H}}_{k} \mathbf{P}_{k \mid k-1}^{(j)} \overline{\mathbf{H}}_{k}^{T}+\overline{\mathbf{R}}_{k}\right)^{-1}, \\
& \mathbf{m}_{k}^{(j)}=\mathbf{m}_{k \mid k-1}^{(j)}+\mathbf{K}_{k}^{(j)}\left(\left[\begin{array}{c}
\mathbf{z}_{1} \\
\vdots \\
\mathbf{z}_{\left|W_{k}\right|}
\end{array}\right]-\overline{\mathbf{H}}_{k} \mathbf{m}_{k \mid k-1}^{(j)}\right) ; \quad \mathbf{P}_{k}^{(j)}=\left(\mathbf{I}-\mathbf{K}_{k}^{(j)} \overline{\mathbf{H}}_{k}\right) \mathbf{P}_{k \mid k-1}^{(j)}, \\
& \left.\overline{\mathbf{H}}_{k}=\left[\begin{array}{c}
\mathbf{H}_{k} \\
\vdots \\
\mathbf{H}_{k}
\end{array}\right]\right\}\left|W_{k}\right| ; \quad \overline{\mathbf{R}}_{k}=\operatorname{blkdiag}(\overbrace{\mathbf{R}_{k}, \ldots, \mathbf{R}_{k}}^{\left|W_{k}\right|}),
\end{aligned}
$$

where I denotes the identity matrix; $p_{D, k}$ has been assumed to be state independent; $\mathbf{H}_{k}$ and $\mathbf{R}_{k}$ denote the observation matrix and the observation noise covariance, respectively; 
$\operatorname{blkdiag}(\cdot)$ denotes block diagonal matrix, the measure in $\langle\cdot, \cdot\rangle$ of $(2.11)$ is discrete, and it defines the summation inner product

$$
\left\langle v_{k \mid k-1}^{J_{k \mid k-1}}, \eta_{W_{k}}\right\rangle=\sum_{l=1}^{J_{k \mid k-1}} \eta_{W_{k}}\left(\mathbf{m}_{k \mid k-1}^{(l)}\right) w_{k \mid k-1}^{(l)}
$$

\section{Convergence of the GM-EPHD and EK-GM-EPHD Filters}

The convergence properties and corresponding proof of the initialization step, prediction step, and pruning and merging step for the GM-EPHD filter are identical to those for point-target GM-PHD filter [19]. The main difficulty and greatest challenge is to prove the convergence for the measurement update step of the filter.

In order to derive the convergence results of the measurement update step for the GM-EPHD filter, the following lemma is first presented.

Consider the following assumptions.

B1: After the prediction step at time $k, v_{k \mid k-1}^{J_{k \mid k-1}}$ converges uniformly to $v_{k \mid k-1}$. In other words, for any given $\varepsilon_{k \mid k-1}>0$ and any bounded measurable function $\varphi \in B\left(\mathbb{R}^{d}\right)$, where $B\left(\mathbb{R}^{d}\right)$ is the set of bounded Borel measurable functions on $\mathbb{R}^{d}$, there is a positive integer $J$ such that

$$
\left|\left\langle v_{k \mid k-1}^{J_{k \mid k-1}}-v_{k \mid k-1}, \varphi\right\rangle\right| \leq \varepsilon_{k \mid k-1}\|\varphi\|_{\infty}
$$

for $J_{k \mid k-1} \geq J$, where $\|\cdot\|_{\infty}$ denotes $\infty$-norm. $\|\varphi\|_{\infty} \triangleq \sup (|\varphi|)$, $\sup (\cdot)$ denotes the supremum.

B2: The clutter intensity $\kappa_{k}\left(\mathbf{z}_{k}\right)=\lambda_{k} c_{k}\left(\mathbf{z}_{k}\right)$ is known a priori.

B3: $\gamma\left(\mathbf{x}_{k}\right) \in C_{b}\left(\mathbb{R}^{d}\right)$, where $C_{b}\left(\mathbb{R}^{d}\right)$ denotes the set of the continuous bounded functions on $\mathbb{R}^{d}$.

Lemma 3.1. Given a partition $\wp_{k}=\left\{W_{1, k}, W_{2, k}, \ldots, W_{n, k}\right\}$ and suppose that assumptions B1-B3 hold, then

$$
\left|\prod_{i=1, \ldots, n} d_{W_{i, k}}^{J_{k \mid k-1}}-\prod_{i=1, \ldots, n} d_{W_{i, k}}\right| \leq \varepsilon_{k \mid k-1} \sum_{j=1, \ldots, n}\left\|\eta_{W_{j, k}}\right\|_{\infty_{i=1, \ldots, n ; i \neq j}}\left(d_{W_{i, k}}^{J_{k \mid k-1}}+d_{W_{i, k}}\right) .
$$

The proof of Lemma 3.1 can be found in Appendix A.

The uniform convergence of the measurement-updated GM-EPHD is now established by Proposition 3.2.

Proposition 3.2. After the measurement update step of the GM-EPHD filter, there exists a real number $a_{k}$, dependent on the number of measurements such that

$$
\left|\left\langle v_{k}^{J_{k}}-v_{k}, \varphi\right\rangle\right| \leq a_{k} \varepsilon_{k \mid k-1}\|\varphi\|_{\infty}
$$

where $a_{k}$ is defined by (B.10). 
The proof of the Proposition 3.2 can be found in Appendix B.

Proposition 3.2 shows that the error for the GM-EPHD corrector converges uniformly to the true EPHD corrector at each stage of the algorithm and the corresponding error bound is also provided. The error tends to zero as the number of Gaussians in the mixture tends to infinity. However, from (B.10), it can be seen that the error bound for the GM-EPHD corrector depends on the number of all partitions of the measurement set. It is quickly realized that as the size of the measurement set increases, the number of possible partitions grows very large. Therefore, the number of Gaussians in the mixture to ensure the asymptotic convergence of the error to a given bound would grow very quickly with the increase of the measurement number.

Now turn to the convergence for the EK-GM-EPHD filter, which is the nonlinear extension of the GM-EPHD filter. Due to the nonlinearity of the extended-target state and observation processes, the EPHD can no longer be represented as a GM. However, the EK-GM-EPHD filter can be adapted to accommodate models with mild nonlinearities. The convergence property and corresponding proof of the prediction step for the EK-GM-EPHD filter are identical to those for point-target EK-GM-PHD filter [19]. We now establish the conditions for uniform convergence of the measurement update step for the EK-GM-EPHD filter.

Proposition 3.3. Suppose that the predicted EK-EPHD is given by the sum of Gaussians

$$
v_{k \mid k-1}^{E K, J_{k \mid k-1}}\left(\mathbf{x}_{k}\right)=\sum_{i=1}^{J_{k \mid k-1}} w_{k \mid k-1}^{(i)} \mathcal{N}\left(\mathbf{x}_{k} \mid \mathbf{m}_{k \mid k-1}^{(i)}, \mathbf{P}_{k \mid k-1}^{(i)}\right),
$$

and the $\phi_{\mathbf{z}_{k}}\left(\mathbf{x}_{k}\right)$ in (2.1) is given by the nonlinear single-measurement single-target equation $\mathbf{z}_{k}=$ $h_{k}\left(\mathbf{x}_{k}, \mathbf{v}_{k}\right)$, where $h_{k}$ is known nonlinear functions and $\mathbf{v}_{k}$ is zero-mean Gaussian measurement noise with covariance $\mathbf{R}_{k}$, then the measurement-updated EK-EPHD approaches the Gaussian sum

$$
v_{k}\left(\mathbf{x}_{k}\right) \rightarrow v_{k}^{E K, J J_{k}}\left(\mathbf{x}_{k}\right)=v_{k}^{N D, E K, J_{k \mid k-1}}\left(\mathbf{x}_{k}\right)+\sum_{\mathfrak{\wp}_{k} \angle Z_{k} W_{k} \in \wp_{k}} v_{k}^{D, E K, J_{k \mid k-1}}\left(\mathbf{x}_{k}, W_{k}\right),
$$

uniformly in $\mathbf{x}_{k}$ and $Z_{k}$ as $\mathbf{P}_{k \mid k-1}^{(i)} \rightarrow 0$ for $i=1, \ldots, J_{k \mid k-1}$, and where

$$
\begin{gathered}
v_{k}^{N D, E K, J_{k \mid k-1}}\left(\mathbf{x}_{k}\right)=\sum_{i=1}^{J_{k \mid k-1}}\left(1-p_{D, k}+e^{-\gamma\left(\mathbf{m}_{k \mid k-1}^{(i)}\right)} p_{D, k}\right) w_{k \mid k-1}^{(i)} \mathcal{N}\left(\mathbf{x}_{k} \mid \mathbf{m}_{k \mid k-1}^{(i)}, \mathbf{P}_{k \mid k-1}^{(i)}\right), \\
v_{k}^{D, E K, J_{k \mid k-1}}\left(\mathbf{x}_{k}, W_{k}\right)=\sum_{i=1}^{J_{k \mid k-1}} \omega_{\wp_{k}}^{E K, J_{k \mid k-1}} \frac{\eta_{W_{k}}^{E K}\left(\mathbf{m}_{k \mid k-1}^{(i)}\right)}{d_{W_{k}}^{E K, J_{k \mid k-1}}} w_{k \mid k-1}^{(i)} N\left(\mathbf{x}_{k} \mid \mathbf{m}_{k}^{(i)}, \mathbf{P}_{k}^{(i)}\right), \\
\omega_{\oint_{k}}^{E K, J_{k \mid k-1}}=\frac{\prod_{W_{k} \in \wp_{k}} d_{W_{k}}^{E K, J_{k \mid k-1}}}{\sum_{\xi_{k}^{\prime} \angle Z_{k}} \prod_{W_{k}^{\prime} \in \xi_{k}^{\prime}} d_{W_{k}^{\prime}}^{E K}} ; \quad d_{W_{k}}^{E K, J_{k \mid k-1}}=\delta_{\left|W_{k}\right|, 1}+\sum_{i=1}^{J_{k \mid k-1}} w_{k \mid k-1}^{(i)} \eta_{W_{k}}^{E K}\left(\mathbf{m}_{k \mid k-1}^{(i)}\right),
\end{gathered}
$$




$$
\begin{aligned}
& \eta_{W_{k}}^{E K}\left(\mathbf{m}_{k \mid k-1}^{(i)}\right)=p_{D, k} e^{-\gamma\left(\mathbf{m}_{k \mid k-1}^{(i)}\right)} \\
& \times \prod_{\mathbf{z}_{k} \in W_{k}} \frac{\gamma\left(\mathbf{m}_{k \mid k-1}^{(i)}\right) \mathcal{N}\left(\mathbf{z}_{k} \mid h_{k}\left(\mathbf{m}_{k \mid k-1}^{(i)}, \mathbf{0}\right), \mathbf{U}_{k}^{(i)} \mathbf{R}_{k}\left(\mathbf{U}_{k}^{(i)}\right)^{T}+\mathbf{H}_{k}^{(i)} \mathbf{P}_{k \mid k-1}^{(i)}\left(\mathbf{H}_{k}^{(i)}\right)^{T}\right)}{\lambda_{k} c_{k}\left(\mathbf{z}_{k}\right)}, \\
& \mathbf{H}_{k}^{(i)}=\left.\frac{\partial h_{k}\left(\mathbf{x}_{k}, \mathbf{0}\right)}{\partial \mathbf{x}_{k}}\right|_{\mathbf{x}_{k}=\mathbf{m}_{k \mid k-1}^{(i)}} ; \quad \mathbf{U}_{k}^{(i)}=\left.\frac{\partial h_{k}\left(\mathbf{m}_{k \mid k-1}^{(i) n}, \mathbf{v}_{k}\right)}{\partial \mathbf{v}_{k}}\right|_{\mathbf{v}_{k}=0} \\
& \mathbf{K}_{k}^{(i)}=\mathbf{P}_{k \mid k-1}^{(i)}\left(\overline{\mathbf{H}}_{k}^{(i)}\right)^{T}\left(\overline{\mathbf{H}}_{k}^{(i)} \mathbf{P}_{k \mid k-1}^{(i)}\left(\overline{\mathbf{H}}_{k}^{(i)}\right)^{T}+\overline{\mathbf{U}}_{k}^{(i)} \mathbf{R}_{k}\left(\overline{\mathbf{U}}_{k}^{(i)}\right)^{T}\right)^{-1} \\
& \mathbf{m}_{k}^{(i)}=\mathbf{m}_{k \mid k-1}^{(i)}+\mathbf{K}_{k}^{(i)}\left(\left[\begin{array}{c}
\mathbf{z}_{1} \\
\vdots \\
\mathbf{z}_{\left|W_{k}\right|}
\end{array}\right]-\left[\begin{array}{c}
h\left(\mathbf{m}_{k \mid k-1}^{(i)}, \mathbf{0}\right) \\
\vdots \\
h\left(\mathbf{m}_{k \mid k-1}^{(i)}, \mathbf{0}\right.
\end{array}\right]\right) ; \mathbf{P}_{k}^{(i)}=\left(\mathbf{I}-\mathbf{K}_{k}^{(i)} \overline{\mathbf{H}}_{k}^{(i)}\right) \mathbf{P}_{k \mid k-1}^{(i)} \\
& \left.\left.\overline{\mathbf{H}}_{k}^{(i)}=\left[\begin{array}{c}
\mathbf{H}_{k}^{(i)} \\
\vdots \\
\mathbf{H}_{k}^{(i)}
\end{array}\right]\right\}\left|W_{k}\right| ; \quad \overline{\mathbf{U}}_{k}^{(i)}=\left[\begin{array}{c}
\mathbf{U}_{k}^{(i)} \\
\vdots \\
\mathbf{U}_{k}^{(i)}
\end{array}\right]\right\}\left|W_{k}\right|
\end{aligned}
$$

The proof of Proposition 3.3 can be found in Appendix C.

From Propositions 3.2 and 3.3, we can obtain that the EK-GM-EPHD corrector uniformly converges to the true EPHD corrector in $\mathbf{x}_{k}$ and $Z_{k}$ under the assumptions that $\mathbf{P}_{k \mid k-1}^{(i)} \rightarrow 0$ for $i=1, \ldots, J_{k \mid k-1}$ and the number of Gaussians in the mixture tends to infinity. These assumptions may be too restrictive or be unrealistic for practical problems, although the EK-GM-EPHD filter have demonstrated its potential for real-world applications. However, Propositions 3.2 and 3.3 give further theoretical justification for the use of the GMEPHD and EK-GM-EPHD filters in ETT problem.

\section{Simulations}

Here we briefly describe the application of the convergence results for the GM-EPHD and EK-GM-EPHD filters to the linear and nonlinear ETT examples.

Example 4.1 (GM-EPHD filter to linear ETT problem). Consider a two-dimensional scenario with an unknown and time varying number of the extended targets observed over the region $[-1000,1000] \times[-1000,1000]$ (in $\mathrm{m}$ ) for a period of $T=45$ time steps. The sampling interval is $\Delta t=1 \mathrm{~s}$. At time $k$, the actual number of the existing extended targets is $n_{k}$ and the state of the $i$ th target is $\mathbf{x}_{i, k}=\left[x_{i, k}, y_{i, k}, \dot{x}_{i, k}, \dot{y}_{i, k}, \ddot{x}_{i, k}, \ddot{y}_{i, k}\right]^{T}\left(i=1, \ldots, n_{k}\right)$. Assume that the process noise $\varpi_{i, k}$ of the $i$ th extended target is independent and identically distributed (IID) zero-mean 
Gaussian white noise with the covariance matrix $\mathbf{Q}_{i, k}$. Then the Markovian transition density of $\mathbf{x}_{i, k}$ could be modeled as

$$
f_{k \mid k-1}\left(\mathbf{x}_{i, k} \mid \mathbf{x}_{i, k-1}\right)=\mathcal{N}\left(\mathbf{x}_{i, k} \mid \mathbf{\Phi}_{i, k} \mathbf{x}_{i, k-1}, \mathbf{Q}_{i, k}\right),
$$

where $\boldsymbol{\Phi}_{i, k}$ is discrete-time evolution matrix. Here $\boldsymbol{\Phi}_{i, k}$ and $\mathbf{Q}_{i, k}$ are given by the constant acceleration model [24], as

$$
\mathbf{\Phi}_{i, k}=\left[\begin{array}{ccc}
1 & \Delta t & \frac{\Delta t^{2}}{2} \\
1 & \frac{\Delta t}{2}
\end{array}\right] \otimes \mathbf{I}_{2} ; \quad \mathbf{Q}_{i, k}=\sigma_{\varpi}^{2}\left[\begin{array}{ccc}
\frac{\Delta t^{4}}{4} & \frac{\Delta t^{3}}{2} & \frac{\Delta t^{2}}{2} \\
\frac{\Delta t^{3}}{2} & \frac{\Delta t^{2}}{2} & \Delta t \\
\frac{\Delta t^{2}}{2} & \Delta t & 1
\end{array}\right] \otimes \mathbf{I}_{2} ; \quad \mathbf{I}_{2}=\left[\begin{array}{ll}
1 & \\
& 1
\end{array}\right]
$$

where " $\otimes$ " denotes the Kronecker product. The parameter $\sigma_{\varpi}$ is the instantaneous standard deviation of the acceleration, given by $\sigma_{\varpi}=0.05 \mathrm{~m} / \mathrm{s}^{2}$.

Note that the objective of this paper is to focus on the convergence analysis for the GMEPHD and EK-GM-EPHD filters, rather than the simulation of the extended-target motions. Therefore, although the proposed evolutions for the extended targets seem to be uncritical and oversimplifying, they will have little effect on the intention of the paper. Readers could be referred to [25] for further discussion on the extended-target motion models. The models proposed in [25] can also be accommodated within the EPHD filter straightforwardly.

At time $k$, the $x$-coordinate and $y$-coordinate measurements of the extended targets are generated by a sensor located at $[0,0]^{T}$. The measurement noise $\mathbf{v}_{k}$ is IID zero-mean Gaussian white noise with covariance matrix $\mathbf{R}_{k}=\operatorname{diag}\left(\sigma_{x}^{2}, \sigma_{y}^{2}\right)$, where $\operatorname{diag}(\cdot)$ denotes the diagonal matrix, $\sigma_{x}$ and $\sigma_{y}$ are, respectively, standard deviations of the $x$-coordinate and $y$-coordinate measurements. In this simulation, they are given as $\sigma_{x}=\sigma_{y}=25 \mathrm{~m}$. The single-measurement single-target likelihood density $\phi_{\mathbf{z}_{k}}\left(\mathbf{x}_{i, k}\right)$ is

$$
\phi_{\mathbf{z}_{k}}\left(\mathbf{x}_{i, k}\right)=\mathcal{N}\left(\mathbf{z}_{k} \mid \mathbf{H}_{k} \mathbf{x}_{i, k}, \mathbf{R}_{k}\right)
$$

where

$$
\mathbf{H}_{k}=\left[\begin{array}{llllll}
1 & 0 & 0 & 0 & 0 & 0 \\
0 & 1 & 0 & 0 & 0 & 0
\end{array}\right]
$$

The detection probability of the sensor is $p_{D, k}\left(\mathbf{x}_{k}\right)=0.95$.

In this simulation, it is assumed that the effect of the shape for each extended target is much smaller than that of the measurement noise. Hence, the shape estimation is not considered here.

At time $k$, the number of the measurements arising from the $i$ th extended target satisfies Poisson distribution with the mean $\gamma\left(\mathbf{x}_{i, k}\right)$. In this simulation, it is given as $\gamma\left(\mathbf{x}_{i, k}\right)=3$ $\left(i=1, \ldots, n_{k}\right)$. 


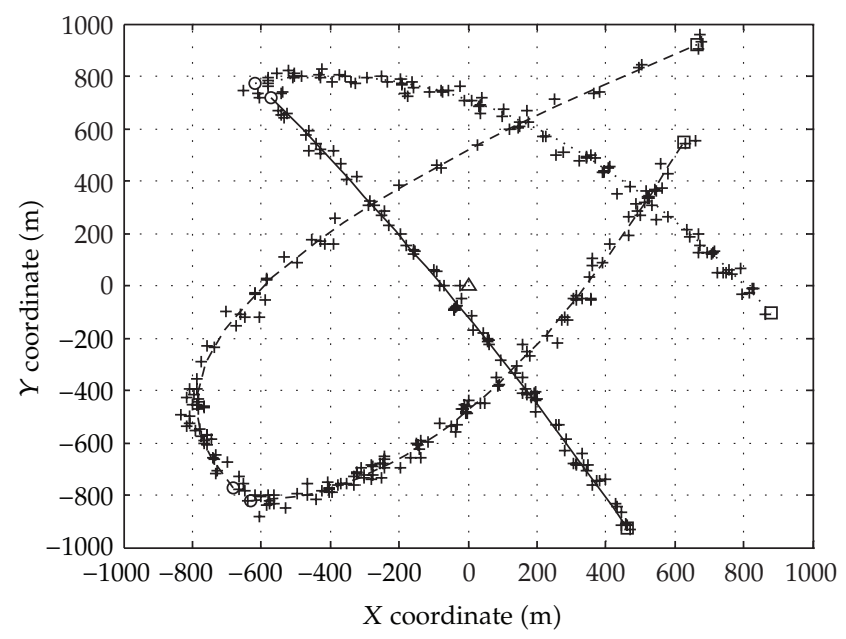

Figure 1: The true trajectories for extended targets and sensor location.

The clutter is modeled as a Poisson RFS with the intensity $\kappa_{k}\left(\mathbf{z}_{k}\right)=\lambda_{k} c_{k}\left(\mathbf{z}_{k}\right)$. In this example, the actual clutter density is $c_{k}\left(\mathbf{z}_{k}\right)=\mathfrak{U}\left(\mathbf{z}_{k}\right)$. It means that the clutter is uniformly distributed over the observation region.

Figure 1 shows the true trajectories for extended targets and sensor location.

In Figure 1, " $\Delta$ " denotes the sensor location, " $\bigcirc$ " denotes the locations at which the extended targets are born, " $\square$ " denotes the locations at which the extended targets die, and " + " denotes the measurements generated by the extended targets. Extended target 1 is born at $1 \mathrm{~s}$ and dies at $25 \mathrm{~s}$. Extended target 2 is born at $1 \mathrm{~s}$ and dies at $30 \mathrm{~s}$. Extended target 3 is born at $10 \mathrm{~s}$ and dies at $35 \mathrm{~s}$. Extended target 4 is born at $20 \mathrm{~s}$ and dies at $45 \mathrm{~s}$.

The intensity of the extended-target birth at time $k$ is modeled as

$$
\beta_{k}\left(\mathbf{x}_{k}\right)=\lambda_{\beta} f_{\beta}\left(\mathbf{x}_{k} \mid \psi_{\beta}\right),
$$

where $\lambda_{\beta}$ is the average number of the extended-target birth per scan, $f_{\beta}\left(\mathbf{x}_{k} \mid \psi_{\beta}\right)$ is the probability density of the new born extended-target state, and $\psi_{\beta}$ is the set of the density parameters. In this example, they are taken as $\lambda_{\beta}=0.05, f_{\beta}\left(\mathbf{x}_{k} \mid \psi_{\beta}\right)=\pi_{\beta}^{1} \mathcal{N}\left(\mathbf{x}_{k} \mid \boldsymbol{\mu}_{\beta}^{1}, \Sigma_{\beta}^{1}\right)+$ $\pi_{\beta}^{2} \mathcal{N}\left(\mathbf{x}_{k} \mid \boldsymbol{\mu}_{\beta^{\prime}}^{2} \Sigma_{\beta}^{2}\right)$, where $\psi_{\beta}=\left\{\pi_{\beta}^{1}, \pi_{\beta}^{2}, \boldsymbol{\mu}_{\beta}^{1}, \boldsymbol{\mu}_{\beta}^{2}, \Sigma_{\beta}^{1}, \Sigma_{\beta}^{2}\right\}, \pi_{\beta}^{1}=\pi_{\beta}^{2}=0.5, \boldsymbol{\mu}_{\beta}^{1}=[-600,750,0,0,0,0]^{T}$, $\boldsymbol{\mu}_{\beta}^{2}=[-650,-800,0,0,0,0]^{T}$, and $\Sigma_{\beta}^{1}=\Sigma_{\beta}^{2}=\operatorname{diag}(400,400,100,100,9,9)$.

The GM-EPHD filter is used to estimate the number and states of the extended targets in the linear ETT problem. We now conduct Monte Carlo (MC) simulation experiments on the same clutter intensity and target trajectories but with independently generated clutter and target-generated measurements in each trial. Via comparing the tracking performance of the GM-EPHD filter in the various number $J_{k}$ of Gaussians in the mixture and in various clutter rate $\lambda_{k}$, the convergence results for the algorithm can be verified to a great extent. For convenience, we assume $J_{k}=J$ and $\lambda_{k}=\lambda$ at each time step. Assumptions B2-B3 are satisfied in this example. So, the GM-EPHD filter uniformly converges to the ground truth.

The standard deviation of the estimated cardinality distribution and the optimal subpattern assignment (OSPA) multitarget miss distance [26] of order $p=2$ with cutoff 
Table 1: Time averaged standard deviation of the estimated cardinality distribution and time averaged OSPA $(\mathrm{m})$ from the GM-EPHD filter in various $J$ given $\lambda=50$.

\begin{tabular}{lccccc}
\hline Gaussian number $J$ in the mixture & 50 & 100 & 300 & 500 & 700 \\
\hline $\begin{array}{l}\text { Time averaged standard deviation of } \\
\text { the estimated cardinality distribution }\end{array}$ & 2.12 & 1.39 & 0.97 & 0.71 & 0.58 \\
$\begin{array}{l}\text { from the GM-EPHD filter } \\
\begin{array}{l}\text { OSPA (m) from the GM-EPHD filter } \\
\hline\end{array}\end{array}$ & 83.5 & 58.7 & 49.6 & 43.1 & 39.5 \\
\hline
\end{tabular}

Table 2: Time averaged standard deviation of the estimated cardinality distribution and time averaged OSPA $(\mathrm{m})$ from the GM-EPHD filter in various $\lambda$ given $J=700$.

\begin{tabular}{|c|c|c|c|c|c|}
\hline Clutter rate $\lambda$ & 50 & 100 & 200 & 300 & 400 \\
\hline $\begin{array}{l}\text { Time averaged standard deviation of } \\
\text { the estimated cardinality distribution } \\
\text { from the GM-EPHD filter }\end{array}$ & 0.58 & 0.70 & 0.95 & 1.23 & 1.48 \\
\hline OSPA $(m)$ from the GM-EPHD filter & 39.5 & 42.9 & 49.0 & 56.1 & 61.7 \\
\hline
\end{tabular}

$c=100 \mathrm{~m}$, which jointly captures differences in cardinality and individual elements between two finite sets, are used to evaluate the performance of the method. Given the clutter rate $\lambda=50$, Table 1 shows the time averaged standard deviation of the estimated cardinality distribution and the time averaged OSPA from the GM-EPHD filter in various $J$ via 200 MC simulation experiments.

Table 1 shows that both the standard deviation of the estimated cardinality distribution and OSPA decrease with the increase of the Gaussian number $J$ in the mixture. This phenomenon can be reasonably explained by the convergence results derived in this paper. First, according to Proposition 3.2, the error of the GM-EPHD decreases as $J$ increases; then, the more precise estimates of the multitarget number and states can be derived from the more precise GM-EPHD, which eventually leads to the results presented in Table 1.

Given $J=700$, Table 2 shows the time averaged standard deviation of the estimated cardinality distribution and the time averaged OSPA from the GM-EPHD filter in various clutter rate $\mathcal{\lambda}$ via $200 \mathrm{MC}$ simulation experiments. Obviously, the number of the measurements collected at each time step increases with the increase of $\lambda$.

From Table 2, it can be seen that the errors of the multitarget number and state estimates from the GM-EPHD filter grow significantly with the increase of $\lambda$. A reasonable explanation for this is that the partition operation included in (B.10) leads that the error bound of the GM-EPHD corrector grows very quickly with the increase of the measurement number. Therefore, Table 2 consists with the convergence results established by Proposition 3.2, too.

Example 4.2 (EK-GM-EPHD filter to nonlinear ETT problem). The experiment settings are the same as those of Example 4.1 except the single-measurement single-target likelihood density $\phi_{\mathbf{z}_{k}}\left(\mathbf{x}_{i, k}\right)$. The range $r_{k}$ and bearing $\theta_{k}$ measurements of the extended targets are generated with the noise covariance matrix $\mathbf{R}_{k}=\operatorname{diag}\left(\sigma_{r}^{2}, \sigma_{\theta}^{2}\right)$, where $\sigma_{r}$ and $\sigma_{\theta}$ are, respectively, standard deviations of the range and bearing measurements. In this simulation, they are given as $\sigma_{r}=25 \mathrm{~m}$ and $\sigma_{\theta}=0.025 \mathrm{rad}$. The $\phi_{\mathbf{z}_{k}}\left(\mathbf{x}_{i, k}\right)$ becomes

$$
\phi_{\mathbf{z}_{k}}\left(\mathbf{x}_{i, k}\right)=\mathcal{N}\left(\mathbf{z}_{k} \mid h_{k}\left(\mathbf{x}_{i, k}\right), \mathbf{R}_{k}\right),
$$


Table 3: Time averaged standard deviation of the estimated cardinality distribution and time averaged OSPA (m) from the EK-GM-EPHD filter in various $J$ given $\lambda=50$.

\begin{tabular}{lccccc}
\hline Gaussian number $J$ in the mixture & 50 & 100 & 300 & 500 & 700 \\
$\begin{array}{l}\text { Time averaged standard deviation of } \\
\text { the estimated cardinality distribution }\end{array}$ & 3.15 & 2.29 & 1.77 & 1.21 & 0.76 \\
$\begin{array}{l}\text { from the EK-GM-EPHD filter } \\
\text { OSPA (m) from the EK-GM-EPHD filter }\end{array}$ & 93.2 & 86.7 & 75.3 & 54.6 & 43.9 \\
\hline
\end{tabular}

Table 4: Time averaged standard deviation of the estimated cardinality distribution and time averaged OSPA (m) from the EK-GM-EPHD filter in various $\lambda$ given $J=700$.

\begin{tabular}{|c|c|c|c|c|c|}
\hline Clutter rate $\lambda$ & 50 & 100 & 200 & 300 & 400 \\
\hline $\begin{array}{l}\text { Time averaged standard deviation of } \\
\text { the estimated cardinality distribution } \\
\text { from the EK-GM-EPHD filter }\end{array}$ & 0.76 & 0.92 & 1.29 & 1.61 & 1.92 \\
\hline OSPA $(m)$ from the EK-GM-EPHD filter & 43.9 & 48.1 & 55.8 & 67.8 & 79.5 \\
\hline
\end{tabular}

where

$$
h_{k}\left(\mathbf{x}_{i, k}\right)=\left[\sqrt{x_{i, k}^{2}+y_{i, k}^{2}} \arctan \frac{y_{i, k}}{x_{i, k}}\right]^{T}
$$

The EK-GM-EPHD filter is used to estimate the number and states of the extended targets in the nonlinear ETT problem. Given $\lambda=50$, Table 3 shows the time averaged standard deviation of the estimated cardinality distribution and the time averaged OSPA from the EKGM-EPHD filter in various $J$ via $200 \mathrm{MC}$ simulation experiments while, given $J=700$, Table 4 shows the time averaged standard deviation of the estimated cardinality distribution and the time averaged OSPA from the EK-GM-EPHD filter in various $\lambda$ via 200 MC simulation experiments.

As expected, Tables 3 and 4, respectively, show that the errors of the multitarget number and state estimates from the EK-GM-EPHD filter decrease with the increase of $J$ and increase with the increase of $\lambda$. These consist with the convergence results established by Propositions 3.2 and 3.3. In addition, comparing Tables 1 and 2 with Tables 3 and 4, it can be seen that the errors from the EK-GM-EPHD filter are obviously larger than the errors from the GM-EPHD filter given the same $J$ and $\lambda$. The additional errors from the EK-GM-EPHD filter are caused by the reason that the condition $\mathbf{P}_{k \mid k-1}^{(i)} \rightarrow 0$ for $i=1, \ldots, J_{k \mid k-1}$ in Proposition 3.3 is very difficult to approach in this example.

\section{Conclusions and Future Work}

This paper shows that the recently proposed GM-EPHD filter converges uniformly to the true EPHD filter as the number of Gaussians in the mixture tends to infinity. Proofs of uniform convergence are also derived for the EK-GM-EPHD filter. Since the GM-EPHD corrector equations involve with the partition operation that grows very quickly with the increase of the measurement number, the future work is focused on studying the computationally 
tractable approximation for it and providing the convergence results and error bounds for the approximate GM-EPHD corrector.

\section{Appendices}

\section{A. Proof of Lemma 3.1}

We have known that $\gamma\left(\mathbf{x}_{k}\right) \in C_{b}\left(\mathbb{R}^{d}\right), \phi_{\mathbf{z}_{k}}\left(\mathbf{x}_{k}\right)=\mathcal{N}\left(\mathbf{z}_{k} \mid \mathbf{H}_{k} \mathbf{x}_{k}, \mathbf{R}_{k}\right) \in C_{b}\left(\mathbb{R}^{d}\right), \lambda_{k} c_{k}\left(\mathbf{z}_{k}\right)$ is known a priori and $0 \leq p_{D, k} \leq 1$ according to assumptions A1-A6 in [12] and assumptions B1-B3. So, from (2.3) we get $\eta_{W_{k}}\left(\mathbf{x}_{k}\right) \in C_{b}\left(\mathbb{R}^{d}\right)$. In addition, by (2.4) and (2.11) and the definition of $\infty$-norm, we have $d_{W_{k}} \geq 0, d_{W_{k}}^{J_{k \mid k-1}} \geq 0$, and $\left\|\eta_{W_{k}}\right\|_{\infty} \geq 0$ because of the facts that $v_{k \mid k-1}\left(\mathbf{x}_{k}\right) \geq 0$, $v_{k \mid k-1}^{J_{k \mid k-1}}\left(\mathbf{x}_{k}\right) \geq 0$ and $\eta_{W_{k}}\left(\mathbf{x}_{k}\right) \geq 0$.

For the initial induction step, assume $n=1$. In this case, from (3.1) we get

$$
\left|d_{W_{1, k}}^{J_{k \mid k-1}}-d_{W_{1, k}}\right|=\left|\left\langle v_{k \mid k-1}^{J_{k \mid k-1}}, \eta_{W_{1, k}}\right\rangle-\left\langle v_{k \mid k-1}, \eta_{W_{1, k}}\right\rangle\right|=\left|\left\langle v_{k \mid k-1}^{J_{k \mid k-1}}-v_{k \mid k-1}, \eta_{W_{1, k}}\right\rangle\right| \leq \varepsilon_{k \mid k-1}\left\|\eta_{W_{1, k}}\right\|_{\infty} .
$$

In the case of $n=2$, by the triangle inequality and (A.1), we have

$$
\begin{aligned}
\left|d_{W_{1, k}}^{J_{k \mid k-1}} d_{W_{2, k}}^{J_{k \mid k-1}}-d_{W_{1, k}} d_{W_{2, k}}\right| & =\left|\frac{d_{W_{2, k}}^{J_{k \mid k-1}}+d_{W_{2, k}}}{2}\left(d_{W_{1, k}}^{J_{k \mid k-1}}-d_{W_{1, k}}\right)+\frac{d_{W_{2, k}}^{J_{k \mid k-1}}-d_{W_{2, k}}}{2}\left(d_{W_{1, k}}^{J_{k \mid k-1}}+d_{W_{1, k}}\right)\right| \\
& \leq \frac{d_{W_{2, k}}^{J_{k \mid k-1}}+d_{W_{2, k}}}{2}\left|d_{W_{1, k}}^{J_{k \mid k-1}}-d_{W_{1, k}}\right|+\left|d_{W_{2, k}}^{J_{k \mid k-1}}-d_{W_{2, k}}\right| \frac{d_{W_{1, k}}^{J_{k \mid k-1}}+d_{W_{1, k}}}{2} \\
& \leq \varepsilon_{k \mid k-1}\left\|\eta_{W_{1, k}}\right\|_{\infty} \frac{d_{W_{2, k}}^{J_{k \mid k-1}}+d_{W_{2, k}}}{2}+\varepsilon_{k \mid k-1}\left\|\eta_{W_{2, k}}\right\|_{\infty} \frac{d_{W_{1, k}}^{J_{k \mid k-1}}+d_{W_{1, k}}}{2} .
\end{aligned}
$$

Since $\varepsilon_{k \mid k-1}>0, d_{W_{k}} \geq 0, d_{W_{k}}^{J_{k \mid k-1}} \geq 0$ and $\left\|\eta_{W_{k}}\right\|_{\infty} \geq 0$, (A.2) becomes

$$
\left|d_{W_{1, k}}^{J_{k \mid k-1}} d_{W_{2, k}}^{J_{k \mid k-1}}-d_{W_{1, k}} d_{W_{2, k}}\right| \leq \varepsilon_{k \mid k-1}\left(\left\|\eta_{W_{1, k}}\right\|_{\infty}\left(d_{W_{2, k}}+d_{W_{2, k}}^{J_{k \mid k-1}}\right)+\left\|\eta_{W_{2, k}}\right\|_{\infty}\left(d_{W_{1, k}}+d_{W_{1, k}}^{J_{k \mid k-1}}\right)\right) .
$$


Assume that we have established (3.2) for $i=1, \ldots, n$. We are to establish (3.2) for $i=1, \ldots, n+1$. Using the triangle inequality and (A.1), we get

$$
\begin{aligned}
& \left|\prod_{i=1, \ldots, n+1} d_{W_{i, k}}^{J_{k \mid k-1}}-\prod_{i=1, \ldots, n+1} d_{W_{i, k}}\right| \\
& =\left|\frac{d_{W_{n+1, k}}+d_{W_{n+1, k}}^{J_{k \mid k-1}}}{2}\left(\prod_{i=1, \ldots, n} d_{W_{i, k}}^{J_{k \mid k-1}}-\prod_{i=1, \ldots, n} d_{W_{i, k}}\right)+\frac{d_{W_{n+1, k}}^{J_{k \mid k-1}}-d_{W_{n+1, k}}}{2}\left(\prod_{i=1, \ldots, n} d_{W_{i, k}}^{J_{k \mid k-1}}+\prod_{i=1, \ldots, n} d_{W_{i, k}}\right)\right| \\
& \leq \frac{d_{W_{n+1, k}}+d_{W_{n+1, k}}^{J_{k \mid k-1}}}{2} \prod_{i=1, \ldots, n} d_{W_{i, k}}^{J_{k \mid k-1}}-\prod_{i=1, \ldots, n} d_{W_{i, k}}|+| \frac{d_{W_{n+1, k}}^{J_{k \mid k-1}}-d_{W_{n+1, k}}}{2} \mid\left(\prod_{i=1, \ldots, n} d_{W_{i, k}}^{J_{k \mid k-1}}+\prod_{i=1, \ldots, n} d_{W_{i, k}}\right) \\
& \leq \varepsilon_{k \mid k-1} \frac{d_{W_{n+1, k}}+d_{W_{n+1, k}}^{J_{k \mid k-1}}}{2} \sum_{j=1, \ldots, n}\left\|\eta_{W_{j, k}}\right\|_{\infty} \prod_{i=1, \ldots, n, i \neq j}\left(d_{W_{i, k}}^{J_{k \mid k-1}}+d_{W_{i, k}}\right) \\
& \quad+\varepsilon_{k \mid k-1} \frac{\left\|\eta_{W_{n+1, k}}\right\|_{\infty}}{2}\left(\prod_{i=1, \ldots, n} d_{W_{i, k}}^{J_{k \mid k-1}}+\prod_{i=1, \ldots, n} d_{W_{i, k}}\right) .
\end{aligned}
$$

Since $\varepsilon_{k \mid k-1}>0, d_{W_{k}} \geq 0, d_{W_{k}}^{J_{k \mid k-1}} \geq 0$ and $\left\|\eta_{W_{k}}\right\|_{\infty} \geq 0$, (A.4) becomes

$$
\begin{aligned}
& \prod_{i=1, \ldots, n+1} d_{W_{i, k}}^{J_{k \mid k-1}}-\prod_{i=1, \ldots, n+1} d_{W_{i, k}} \mid \\
& \leq \varepsilon_{k \mid k-1} \sum_{j=1, \ldots, n}\left\|\eta_{W_{j, k}}\right\|_{\infty_{i=1, \ldots, n+1 ; i \neq j}}\left(d_{W_{i, k}}^{J_{k \mid k-1}}+d_{W_{i, k}}\right)+\varepsilon_{k \mid k-1}\left\|\eta_{W_{n+1, k}}\right\|_{\infty}\left(\prod_{i=1, \ldots, n} d_{W_{i, k}}^{J_{k \mid k-1}}+\prod_{i=1, \ldots, n} d_{W_{i, k}}\right) \\
& \leq \varepsilon_{k \mid k-1} \sum_{j=1, \ldots, n}\left\|\eta_{W_{j, k}}\right\|_{\infty_{i=1, \ldots, n+1 ; i \neq j}}\left(d_{W_{i, k}}^{J_{k \mid k-1}}+d_{W_{i, k}}\right)+\varepsilon_{k \mid k-1}\left\|\eta_{W_{n+1, k}}\right\|_{\infty} \prod_{i=1, \ldots, n}\left(d_{W_{i, k}}^{J_{k \mid k-1}}+d_{W_{i, k}}\right) \\
& =\varepsilon_{k \mid k-1} \sum_{j=1, \ldots, n+1}\left\|\eta_{W_{j, k}}\right\|_{\infty} \prod_{i=1, \ldots, n+1 ; i \neq j}\left(d_{W_{i, k}}^{J_{k \mid k-1}}+d_{W_{i, k}}\right),
\end{aligned}
$$

and this closes the inductive step. This completes the proof. 


\section{B. Proof of Proposition 3.2}

By the EPHD corrector equations, (2.2), and the triangle inequality, we get

$$
\begin{aligned}
& \left|\left\langle v_{k}^{J_{k}}-v_{k}, \varphi\right\rangle\right| \\
& =\mid\left\langle\left[1-p_{D, k}+e^{-\gamma} p_{D, k}+\sum_{\wp_{k} \angle Z_{k}} \omega_{\wp_{k}}^{J_{k \mid k-1}} \sum_{W_{k} \in \wp_{k}} \frac{\eta_{W_{k}}}{d_{W_{k}}^{J_{k \mid k-1}}}\right] v_{k \mid k-1}^{J_{k \mid k-1}}, \varphi\right\rangle \\
& -\left\langle\left[1-p_{D, k}+e^{-\gamma} p_{D, k}+\sum_{\wp_{k} \angle Z_{k}} \omega_{\wp_{k}} \sum_{W_{k} \in \mathfrak{\wp}_{k}} \frac{\eta_{W_{k}}}{d_{W_{k}}}\right] v_{k \mid k-1}, \varphi\right\rangle \mid \\
& =\left|\begin{array}{c}
\left(\left\langle v_{k \mid k-1}^{J_{k \mid k-1}}, \varphi\left(1-p_{D, k}+e^{-\gamma} p_{D, k}\right)\right\rangle-\left\langle v_{k \mid k-1}, \varphi\left(1-p_{D, k}+e^{-\gamma} p_{D, k}\right)\right\rangle\right) \\
+\sum_{\wp_{k} \angle Z_{k} W_{k} \in \wp_{k}}\left(\frac{\omega_{\wp_{k}}^{J_{k \mid k-1}}\left\langle v_{k \mid k-1}^{J_{k \mid k-1}}, \varphi \eta_{W_{k}}\right\rangle}{d_{W_{k}}^{J_{k \mid k-1}}}-\frac{\omega_{\mathfrak{\wp}_{k}}\left\langle v_{k \mid k-1}, \varphi \eta_{W_{k}}\right\rangle}{d_{W_{k}}}\right)
\end{array}\right| \\
& \leq\left|\left\langle v_{k \mid k-1}^{J_{k \mid k-1}}-v_{k \mid k-1}, \varphi\left(1-p_{D, k}+e^{-\gamma} p_{D, k}\right)\right\rangle\right| \\
& +\sum_{\wp_{k} \angle Z_{k} W_{k} \in \wp_{k}}\left(\left|\frac{\omega_{\wp_{k}}^{J_{k \mid k-1}}\left\langle v_{k \mid k-1}^{J_{k \mid k-1}}, \varphi \eta_{W_{k}}\right\rangle}{d_{W_{k}}^{J_{k \mid k-1}}}-\frac{\omega_{\wp_{k}}\left\langle v_{k \mid k-1}^{J_{k \mid k-1}}, \varphi \eta_{W_{k}}\right\rangle}{d_{W_{k}}}\right|\right. \\
& \left.+\left|\frac{\omega_{\mathfrak{f}_{k}}\left\langle v_{k \mid k-1}^{J_{k \mid k-1}}, \varphi \eta_{W_{k}}\right\rangle}{d_{W_{k}}}-\frac{\omega_{\mathfrak{\wp}_{k}}\left\langle v_{k \mid k-1}, \varphi \eta_{W_{k}}\right\rangle}{d_{W_{k}}}\right|\right) .
\end{aligned}
$$

By (3.1), the second term in the summation of (B.1) is

$$
\begin{aligned}
\left|\frac{\omega_{\wp_{k}}\left\langle v_{k \mid k-1}^{J_{k \mid k-1}}, \varphi \eta_{W_{k}}\right\rangle}{d_{W_{k}}}-\frac{\omega_{\wp_{k}}\left\langle v_{k \mid k-1}, \varphi \eta_{W_{k}}\right\rangle}{d_{W_{k}}}\right| & =\frac{\omega_{\wp_{k}}\left|\left\langle v_{k \mid k-1}^{J_{k \mid k-1}}-v_{k \mid k-1}, \varphi \eta_{W_{k}}\right\rangle\right|}{d_{W_{k}}} \\
& \leq \frac{\omega_{\wp_{k}} \varepsilon_{k \mid k-1}\left\|\varphi \eta_{W_{k}}\right\|_{\infty}}{d_{W_{k}}} .
\end{aligned}
$$


Journal of Applied Mathematics

Using the triangle inequality, the first term in the summation of (B.1) is

$$
\begin{aligned}
& \left|\frac{\omega_{\wp_{k}}^{J_{k \mid k-1}}\left\langle v_{k \mid k-1}^{J_{k \mid k-1}}, \varphi \eta_{W_{k}}\right\rangle}{d_{W_{k}}^{J_{k \mid k-1}}}-\frac{\omega_{\wp_{k}}\left\langle v_{k \mid k-1}^{J_{k \mid k-1}}, \varphi \eta_{W_{k}}\right\rangle}{d_{W_{k}}}\right| \\
& =\frac{\left\langle v_{k \mid k-1}^{J_{k \mid k-1}}, \varphi \eta_{W_{k}}\right\rangle\left|d_{W_{k}} \omega_{\wp_{k}}^{J_{k \mid k-1}}-d_{W_{k}}^{J_{k \mid k-1}} \omega_{\wp_{k}}\right|}{d_{W_{k}}^{J_{k \mid k-1}} d_{W_{k}}}
\end{aligned}
$$

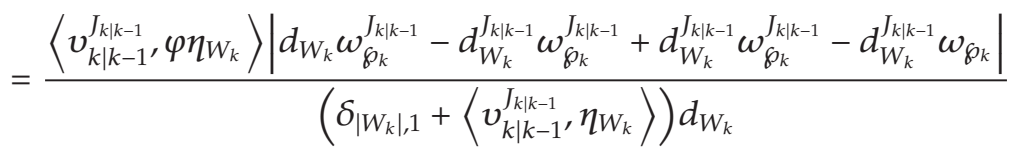

$$
\begin{aligned}
& \leq \frac{\left\langle v_{k \mid k-1}^{J_{k \mid k-1}}, \eta_{W_{k}}\right\rangle\|\varphi\|_{\infty}\left|\omega_{\mathfrak{\wp}_{k}}^{J_{k \mid k-1}}\left(d_{W_{k}}-d_{W_{k}}^{J_{k \mid k-1}}\right)+d_{W_{k}}^{J_{k \mid k-1}}\left(\omega_{\mathfrak{\wp}_{k}}^{J_{k \mid k-1}}-\omega_{\mathfrak{\xi}_{k}}\right)\right|}{\left\langle v_{k \mid k-1}^{J_{k \mid k-1}}, \eta_{W_{k}}\right\rangle d_{W_{k}}} \\
& \leq \frac{\|\varphi\|_{\infty}\left(\omega_{\mathscr{\wp}_{k}}^{J_{k \mid k-1}}\left|d_{W_{k}}-d_{W_{k}}^{J_{k \mid k-1}}\right|+d_{W_{k}}^{J_{k \mid k-1}}\left|\omega_{\mathfrak{\wp}_{k}}^{J_{k \mid k-1}}-\omega_{\wp_{k}}\right|\right)}{d_{W_{k}}} .
\end{aligned}
$$

Using the triangle inequality again for the term $\left|\omega_{\delta_{\mathrm{k}}}^{J_{k \mid k-1}}-\omega_{\wp_{\mathrm{k}}}\right|$ in the numerator of (B.3), we get

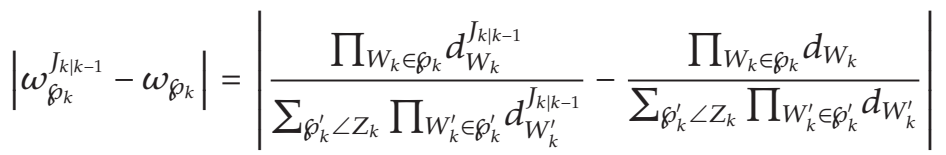

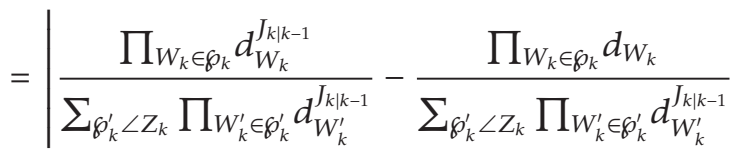

$$
\begin{aligned}
& +\frac{\prod_{W_{k} \in \mathfrak{\wp}_{k}} d_{W_{k}}}{\sum_{\mathfrak{\wp}_{k}^{\prime}} \angle Z_{k} \prod_{W_{k}^{\prime} \in \mathfrak{\delta}_{k}^{\prime}} d_{W_{k}^{\prime}}^{J_{k \mid k-1}}}-\frac{\prod_{W_{k} \in \mathfrak{\wp}_{k}} d_{W_{k}}}{\sum_{\mathfrak{\wp}_{k}^{\prime} \angle Z_{k}} \prod_{W_{k}^{\prime} \in \mathfrak{\xi}_{k}^{\prime}} d_{W_{k}^{\prime}}} \mid
\end{aligned}
$$

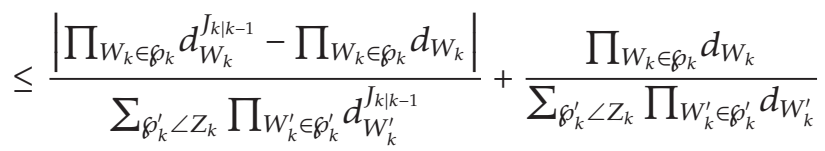

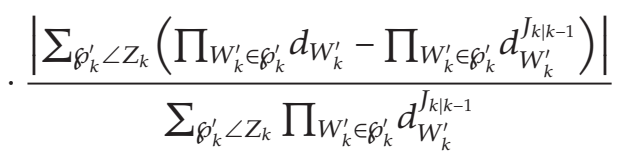

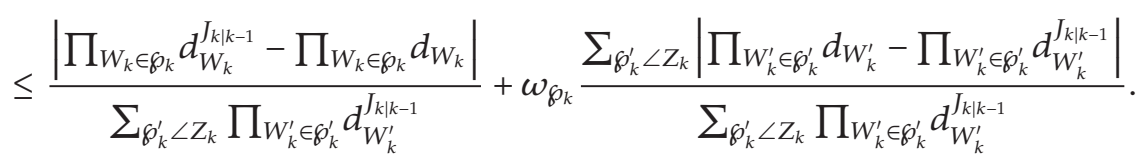


Using Lemma 3.1, we get

$$
\left|\prod_{W_{k} \in \mathfrak{\wp}_{k}} d_{W_{k}}-\prod_{W_{k} \in \mathfrak{q}_{k}} d_{W_{k}}^{J_{k \mid k-1}}\right| \leq \varepsilon_{k \mid k-1} \sum_{W_{k} \in \mathfrak{\wp}_{k}}\left\|\eta_{W_{k}}\right\|_{\infty} \prod_{V_{k} \in \mathfrak{\wp}_{k}-W_{k}}\left(d_{V_{k}}+d_{V_{k}}^{J_{k \mid k-1}}\right),
$$

where $\wp_{k}-W_{k}$ denotes the complement of $W_{k}$ in $\wp_{k}$.

Then, (B.4) can be rewritten as

$$
\left|\omega_{\wp_{k}}^{J_{k \mid k-1}}-\omega_{\wp_{k}}\right| \leq \varepsilon_{k \mid k-1} \rho_{k}
$$

where

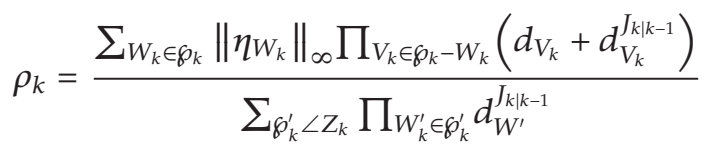

$$
\begin{aligned}
& +\frac{\omega_{\mathscr{\wp}_{k}} \sum_{\mathscr{\wp}_{k}^{\prime} \angle Z_{k}} \sum_{W_{k}^{\prime} \in \mathscr{\xi}_{k}^{\prime}}\left\|\eta_{W_{k}^{\prime}}\right\|_{\infty} \prod_{V_{k}^{\prime} \in \mathfrak{\wp}_{k}^{\prime}-W_{k}^{\prime}}\left(d_{V_{k}^{\prime}}+d_{V_{k}^{\prime}}^{J_{k \mid k-1}}\right)}{\sum_{\mathfrak{\wp}_{k}^{\prime} \angle Z_{k}} \prod_{W_{k}^{\prime} \in \mathfrak{\wp}_{k}^{\prime}} d_{W^{\prime}}^{J_{k \mid k-1}}} .
\end{aligned}
$$

Substitute (A.1) and (B.6) into (B.3),

$$
\left|\frac{\omega_{\wp_{k}}^{J_{k \mid k-1}}\left\langle v_{k \mid k-1}^{J_{k \mid k-1}}, \varphi \eta_{W_{k}}\right\rangle}{d_{W_{k}}^{J_{k}}}-\frac{\omega_{\xi_{k}}\left\langle v_{k \mid k-1}^{J_{k \mid k-1}}, \varphi \eta_{W_{k}}\right\rangle}{d_{W_{k}}}\right| \leq \varepsilon_{k \mid k-1} \frac{\|\varphi\|_{\infty}\left(\omega_{\wp_{k}}^{J_{k \mid k-1}}\left\|\eta_{W_{k}}\right\|_{\infty}+d_{W_{k}}^{J_{k \mid k-1}} \rho_{k}\right)}{d_{W_{k}}} .
$$

Substituting (3.1), (B.2), and (B.8) into (B.1), we have

$$
\begin{aligned}
\left|\left\langle v_{k}^{J_{k \mid k-1}}-v_{k}, \varphi\right\rangle\right| \leq \varepsilon_{k \mid k-1}\|\varphi\|_{\infty} & \left\|1-p_{D, k}+e^{-\gamma} p_{D, k}\right\|_{\infty} \\
& \left.+\sum_{\wp_{k} \angle Z_{k} W_{k} \in \wp_{k}}\left(\frac{\omega_{\wp_{k}}^{J_{k \mid k-1}}\left\|\eta_{W_{k}}\right\|_{\infty}+d_{W_{k}}^{J_{k \mid k-1}} \rho_{k}}{d_{W_{k}}}+\omega_{\wp_{k}} \frac{\left\|\eta_{W_{k}}\right\|_{\infty}}{d_{W_{k}}}\right)\right) .
\end{aligned}
$$

So that Proposition 3.2 is proved with

$$
a_{k}=\left\|1-p_{D, k}+e^{-\gamma} p_{D, k}\right\|_{\infty}+\sum_{\wp_{k} \angle Z_{k}} \sum_{W_{k} \in \wp_{k}} \frac{\omega_{\wp_{k}}^{J_{k \mid k-1}}\left\|\eta_{W_{k}}\right\|_{\infty}+d_{W_{k}}^{J_{k \mid k-1}} \rho_{k}+\omega_{\mathfrak{\wp}_{k}}\left\|\eta_{W_{k}}\right\|_{\infty}}{d_{W_{k}}} .
$$

This completes the proof. 
Journal of Applied Mathematics

\section{Proof of Proposition 3.3}

Clearly, by the EPHD corrector equations, (2.2)-(2.4), and the predicted EK-EPHD, (3.4), we obtain that the $v_{k}^{N D, E K, J_{k \mid k-1}}\left(\mathbf{x}_{k}\right)$ in (3.5) is a Gaussian sum presented by (3.6). Now turn to the $v_{k}^{D, E K, J_{k \mid k-1}}\left(\mathbf{x}_{k}, W_{k}\right)$ in (3.5). From (2.2), we get

$$
v_{k}^{D, E K, J_{k \mid k-1}}\left(\mathbf{x}_{k}, W_{k}\right)=\omega_{\wp_{k}}^{J_{k \mid k-1}} \frac{\eta_{W_{k}}\left(\mathbf{x}_{k}\right)}{d_{W_{k}}^{k \mid k-1}} v_{k \mid k-1}^{E K, J_{k \mid k-1}}\left(\mathbf{x}_{k}\right)
$$

Consider the term $\eta_{W_{k}}\left(\mathbf{x}_{k}\right) v_{k \mid k-1}^{E K, J_{k \mid k-1}}\left(\mathbf{x}_{k}\right)$ in (C.1). Using the predicted EK-EPHD, (3.4),

$$
\eta_{W_{k}}\left(\mathbf{x}_{k}\right) v_{k \mid k-1}^{E K, J_{k \mid k-1}}\left(\mathbf{x}_{k}\right)=p_{D, k} e^{-\gamma\left(\mathbf{x}_{k}\right)} \prod_{\mathbf{z}_{k} \in W_{k}} \frac{\gamma\left(\mathbf{x}_{k}\right) \phi_{\mathbf{z}_{k}}\left(\mathbf{x}_{k}\right)}{\lambda_{k} c_{k}\left(\mathbf{z}_{k}\right)} \sum_{i=1}^{J_{k \mid k-1}} w_{k \mid k-1}^{(i)} \mathcal{N}\left(\mathbf{x}_{k} \mid \mathbf{m}_{k \mid k-1}^{(i)}, \mathbf{P}_{k \mid k-1}^{(i)}\right) .
$$

And by the result for the EK Gaussian sum filter [17], we get

$$
\begin{aligned}
\eta_{W_{k}}\left(\mathbf{x}_{k}\right) & v_{k \mid k-1}^{E K, J_{k \mid k-1}}\left(\mathbf{x}_{k}\right) \\
\longrightarrow & \sum_{i=1}^{J J_{k \mid k-1}} w_{k \mid k-1}^{(i)} p_{D, k} e^{-\gamma\left(\mathbf{m}_{k \mid k-1}^{(i)}\right)} \\
& \times \prod_{\mathbf{z}_{k} \in W_{k}} \frac{\gamma\left(\mathbf{m}_{k \mid k-1}^{(i)}\right) \mathcal{N}\left(\mathbf{z}_{k} \mid h_{k}\left(\mathbf{m}_{k \mid k-1}^{(i)}, \mathbf{0}\right), \mathbf{U}_{k}^{(i)} \mathbf{R}_{k}\left(\mathbf{U}_{k}^{(i)}\right)^{T}+\mathbf{H}_{k}^{(i)} \mathbf{P}_{k \mid k-1}^{(i)}\left(\mathbf{H}_{k}^{(i)}\right)^{T}\right)}{\lambda_{k} c_{k}\left(\mathbf{z}_{k}\right)} \\
& \times \mathcal{N}\left(\mathbf{x}_{k} \mid \mathbf{m}_{k}^{(i)}, \mathbf{P}_{k}^{(i)}\right) \\
= & \sum_{i=1}^{J_{k \mid k-1}} w_{k \mid k-1}^{(i)} \eta_{W_{k}}^{E K}\left(\mathbf{m}_{k \mid k-1}^{(i)}\right) \mathcal{N}\left(\mathbf{x}_{k} \mid \mathbf{m}_{k}^{(i)}, \mathbf{P}_{k}^{(i)}\right),
\end{aligned}
$$

uniformly as $\mathbf{P}_{k \mid k-1}^{(i)} \rightarrow 0$ for all $i=1, \ldots, J_{k \mid k-1}$, and $\eta_{W_{k}}^{E K}\left(\mathbf{m}_{k \mid k-1}^{(i)}\right), \mathbf{H}_{k}^{(i)}, \mathbf{U}_{k}^{(i)}, \mathbf{m}_{k}^{(i)}, \mathbf{P}_{k}^{(i)}$ are given by (3.9)-(3.13), respectively.

Now consider the terms $\omega_{\hat{\wp}_{k}}^{J_{k \mid k-1}}$ and $d_{W_{k}}^{J_{k \mid k-1}}$ in (C.1). First, using the predicted EK-EPHD, (3.4), the inner product $\left\langle v_{k \mid k-1}^{E K, J_{k \mid k-1}}, \eta_{W_{k}}\right\rangle$ is given by

$$
\begin{aligned}
\left\langle v_{k \mid k-1}^{E K, J_{k \mid k-1}}, \eta_{W_{k}}\right\rangle & =\int \eta_{W_{k}}\left(\mathbf{x}_{k}\right) v_{k \mid k-1}^{E K, J_{k \mid k-1}}\left(\mathbf{x}_{k}\right) d \mathbf{x}_{k} \\
& =p_{D, k} \int e^{-\gamma\left(\mathbf{x}_{k}\right)} \prod_{\mathbf{z}_{k} \in W_{k}} \frac{\gamma\left(\mathbf{x}_{k}\right) \phi_{\mathbf{z}_{k}}\left(\mathbf{x}_{k}\right)}{\lambda_{k} C_{k}\left(\mathbf{z}_{k}\right)} \sum_{i=1}^{J_{k \mid k-1}} w_{k \mid k-1}^{(i)} N\left(\mathbf{x}_{k} \mid \mathbf{m}_{k \mid k-1}^{(i)}, \mathbf{P}_{k \mid k-1}^{(i)}\right) d \mathbf{x}_{k} .
\end{aligned}
$$


And by the result for the EK Gaussian sum filter [17], we get

$$
\begin{aligned}
\left\langle v_{k \mid k-1}^{E K, J_{k \mid k-1}},\right. & \left.\eta_{W_{k}}\right\rangle \\
\longrightarrow & \int_{i=1}^{J_{k \mid k-1}} w_{k \mid k-1}^{(i)} p_{D, k} e^{-\gamma\left(\mathbf{x}_{k}\right)} \\
& \times \prod_{\mathbf{z}_{k} \in W_{k}} \frac{\gamma\left(\mathbf{m}_{k \mid k-1}^{(i)}\right) \mathcal{N}\left(\mathbf{z}_{k} \mid h_{k}\left(\mathbf{m}_{k \mid k-1}^{(i)}, \mathbf{0}\right), \mathbf{U}_{k}^{(i)} \mathbf{R}_{k}\left(\mathbf{U}_{k}^{(i)}\right)^{T}+\mathbf{H}_{k}^{(i)} \mathbf{P}_{k \mid k-1}^{(i)}\left(\mathbf{H}_{k}^{(i)}\right)^{T}\right)}{\lambda_{k} c_{k}\left(\mathbf{z}_{k}\right)} \\
& \times \mathcal{N}\left(\mathbf{x}_{k} \mathbf{m}_{k}^{(i)}, \mathbf{P}_{k}^{(i)}\right) d \mathbf{x}_{k},
\end{aligned}
$$

uniformly as $\mathbf{P}_{k \mid k-1}^{(i)} \rightarrow 0$ for all $i=1, \ldots, J_{k \mid k-1}$.

Changing the order of the summation and integral, (C.5) is equal to

$$
\begin{aligned}
& \left\langle v_{k \mid k-1}^{E K, J_{k \mid k-1}}, \eta_{W_{k}}\right\rangle \\
& \quad \longrightarrow \sum_{i=1}^{J_{k \mid k-1}} \int w_{k \mid k-1}^{(i)} p_{D, k} e^{-\gamma\left(\mathbf{m}_{k \mid k-1}^{(i)}\right)} \\
& \quad \times \prod_{\mathbf{z}_{k} \in W_{k}} \frac{\gamma\left(\mathbf{m}_{k \mid k-1}^{(i)}\right) \mathcal{N}\left(\mathbf{z}_{k} \mid h_{k}\left(\mathbf{m}_{k \mid k-1}^{(i)}, \mathbf{0}\right), \mathbf{U}_{k}^{(i)} \mathbf{R}_{k}\left(\mathbf{U}_{k}^{(i)}\right)^{T}+\mathbf{H}_{k}^{(i)} \mathbf{P}_{k \mid k-1}^{(i)}\left(\mathbf{H}_{k}^{(i)}\right)^{T}\right)}{\lambda_{k} c_{k}\left(\mathbf{z}_{k}\right)} \\
& \quad \times \mathcal{N}\left(\mathbf{x}_{k} \mid \mathbf{m}_{k}^{(i)}, \mathbf{P}_{k}^{(i)}\right) d \mathbf{x}_{k} \\
& =\sum_{i=1}^{J_{k \mid k-1}} w_{k \mid k-1}^{(i)} \eta_{W_{k}}^{E K}\left(\mathbf{m}_{k \mid k-1}^{(i)}\right) \int \mathcal{N}\left(\mathbf{x}_{k} \mid \mathbf{m}_{k}^{(i)}, \mathbf{P}_{k}^{(i)}\right) d \mathbf{x}_{k} \\
& =\sum_{i=1}^{J_{k \mid k-1}} w_{k \mid k-1}^{(i)} \eta_{W_{k}}^{E K}\left(\mathbf{m}_{k \mid k-1}^{(i)}\right) .
\end{aligned}
$$
(C.6).

Then, the expressions of $\omega_{W_{k}}^{E K, J_{k \mid k-1}}$ and $d_{W_{k}}^{E K, J_{k \mid k-1}}$ (see (3.8)) are derived by (2.4) and proof.

Finally, (3.7) is obtained by substituting (3.8) and (C.3) into (C.1). This completes the

\section{Acknowledgments}

This research work was supported by Natural Science Foundation of China (61004087, 61104051, 61104214, and 61005026), China Postdoctoral Science Foundation (20100481338 and 2011M501443), and Fundamental Research Funds for the Central University. 


\section{References}

[1] M. J. Waxmann and O. E. Drummond, "A bibliography of cluster (group) tracking," in Signal and Data Processing of Small Targets, vol. 5428 of Proceedings of SPIE, pp. 551-560, Orlando, Fla, USA.

[2] F. Lian, C. Z. Han, W. F. Liu, X. X. Yan, and H. Y. Zhou, "Sequential Monte Carlo implementation and state extraction of the group probability hypothesis density filter for partly unresolvable group targets-tracking problem," IET Radar, Sonar and Navigation, vol. 4, no. 5, pp. 685-702, 2010.

[3] F. Daum and R. Fitzgerald, "Importance of resolution in multiple-target tracking," in Signal and Data Processing of Small Targets, vol. 2235 of Proceedings of SPIE, pp. 329-338, Orlando, Fla, USA, April 1994.

[4] K. Granstrom, C. Lundquist, and U. Orguner, "Tracking rectangular and elliptical extended targets using laser measurements," in Proceedings of the 14th International Conference on Information Fusion, pp. 1-8, Chicago, Ill, USA, July 2011.

[5] U. Orguner, C. Lundquist, and K. Granstrom, "Extended target tracking with a cardinalized probability hypothesis density filter," in Proceedings of the 14th International Conference on Information Fusion, pp. 1-8, Chicago, Ill, USA, July 2011.

[6] J. W. Koch, "Bayesian approach to extended object and cluster tracking using random matrices," IEEE Transactions on Aerospace and Electronic Systems, vol. 44, no. 3, pp. 1042-1059, 2008.

[7] M. Feldmann and D. Fränken, "Tracking of extended objects and group targets using random matrices-a new approach," in Proceedings of the 11th International Conference on Information Fusion (FUSION '08), pp. 1-8, Cologne, Germany, July 2008.

[8] Y. Bar-Shalom and X. R. Li, Multitarget-Multisensor Tracking: Principles and Techniques, YBS Publishing, Storrs, Conn, USA, 1995.

[9] I. R. Goodman, R. P. S. Mahler, and H. T. Nguyen, Mathematics of Data Fusion, vol. 37 of Theory and Decision Library. Series B: Mathematical and Statistical Methods, Kluwer Academic, Norwood, Mass, USA, 1997.

[10] R. P. S. Mahler, "Multitarget Bayes filtering via first-order multitarget moments," IEEE Transactions on Aerospace and Electronic Systems, vol. 39, no. 4, pp. 1152-1178, 2003.

[11] B. N. Vo, S. Singh, and A. Doucet, "Sequential Monte Carlo methods for multi-target filtering with random finite sets," IEEE Transactions on Aerospace and Electronic Systems, vol. 41, no. 4, pp. 1224-1245, 2005.

[12] B. N. Vo and W. K. Ma, "The Gaussian mixture probability hypothesis density filter," IEEE Transactions on Signal Processing, vol. 54, no. 11, pp. 4091-4104, 2006.

[13] D. Clark and S. Godsill, "Group target tracking with the gaussian mixture probability hypothesis density filter," in Proceedings of the International Conference on Intelligent Sensors, Sensor Networks and Information Processing (ISSNIP '07), pp. 149-154, Melbourne, Australia, December 2007.

[14] K. Gilholm, S. Godsill, S. Maskell, and D. Salmond, "Poisson models for extended target and group tracking," in Signal and Data Processing of Small Targets, vol. 5913 of Proceedings of the SPIE, pp. 1-12, San Diego, Calif, USA, August 2005.

[15] R. Mahler, "PHD filters for nonstandard targets, I: extended targets," in Proceedings of the 12th International Conference on Information Fusion (FUSION '09), pp. 915-921, Seattle, Wash, USA, July 2009.

[16] K. Granström, C. Lundquist, and U. Orguner, "A Gaussian mixture PHD filter for extended target tracking," in Proceedings of the 13th Conference on Information Fusion (FUSION '10), pp. 1-8, Edinburgh, UK, July 2010.

[17] B. D. Anderson and J. B. Moore, Optimal Filtering, Prentice-Hall, Englewood Cliffs, NJ, USA, 1979.

[18] D. E. Clark and J. Bell, "Convergence results for the particle PHD filter," IEEE Transactions on Signal Processing, vol. 54, no. 7, pp. 2652-2661, 2006.

[19] D. Clark and B.-N. Vo, "Convergence analysis of the Gaussian mixture PHD filter," IEEE Transactions on Signal Processing, vol. 55, no. 4, pp. 1204-1212, 2007.

[20] P. Cholamjiak, S. Suantai, and Y. J. Cho, "Strong convergence to solutions of generalized mixed equilibrium problems with applications," Journal of Applied Mathematics, vol. 2012, Article ID 308791, 18 pages, 2012.

[21] V. Colao, "On the convergence of iterative processes for generalized strongly asymptotically $\varphi$ pseudocontractive mappings in Banach spaces," Journal of Applied Mathematics, vol. 2012, Article ID 563438, 18 pages, 2012.

[22] G. Gu, S. Wang, and Y. J. Cho, "Strong convergence algorithms for hierarchical fixed points problems and variational inequalities," Journal of Applied Mathematics, vol. 2011, Article ID 164978, 17 pages, 2011. 
[23] R. Mahler, Statistical Multisource Multitarget Information Fusion, Artech House, Norwood, Mass, USA, 2007.

[24] X. R. Li and V. P. Jikov, "A survey of maneuvering target tracking: dynamic models," in Signal and Data Processing of Small Targets, vol. 4048 of Proceedings of SPIE, pp. 212-235, Orlando, Fla, USA, April 2000.

[25] D. J. Salmond and N. J. Gordon, "Group and extended object tracking," IEE Colloquium on Target Tracking, vol. 16, pp. 1-4, 1999.

[26] D. Schuhmacher, B.-T. Vo, and B.-N. Vo, "A consistent metric for performance evaluation of multiobject filters," IEEE Transactions on Signal Processing, vol. 56, no. 8, pp. 3447-3457, 2008. 


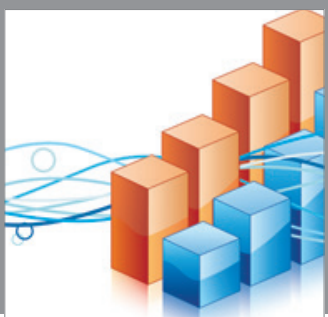

Advances in

Operations Research

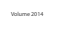

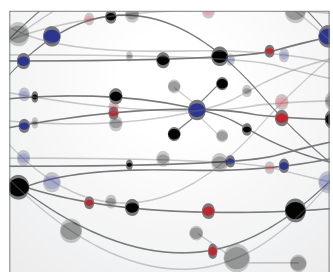

\section{The Scientific} World Journal
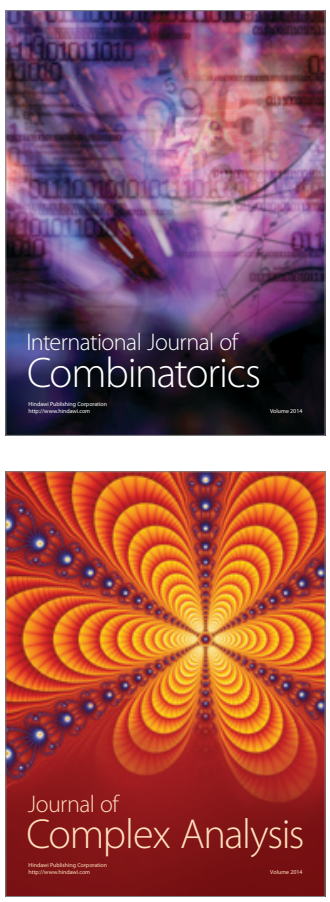

International Journal of

Mathematics and

Mathematical

Sciences
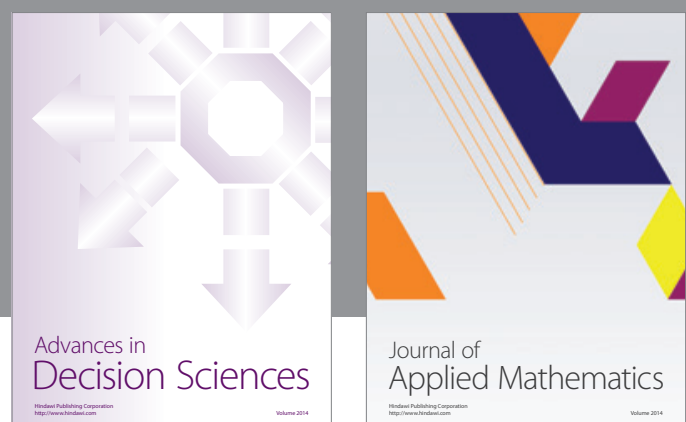

Journal of

Applied Mathematics
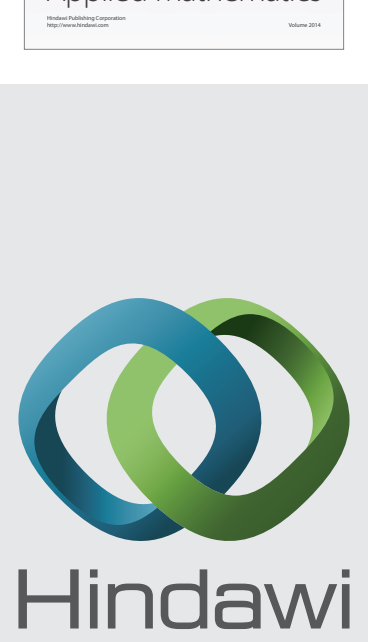

Submit your manuscripts at http://www.hindawi.com
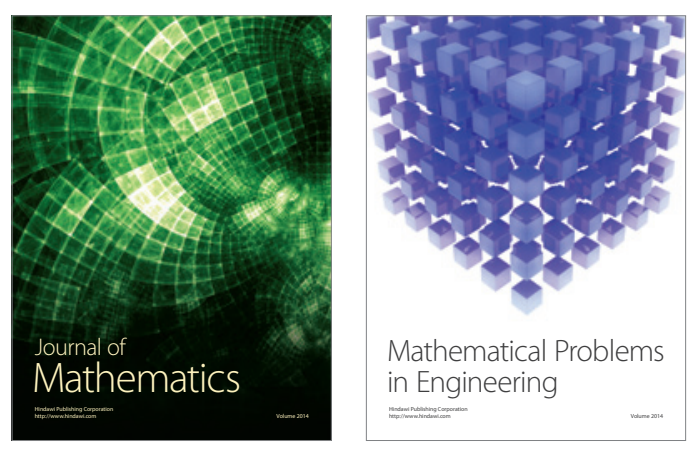

Mathematical Problems in Engineering
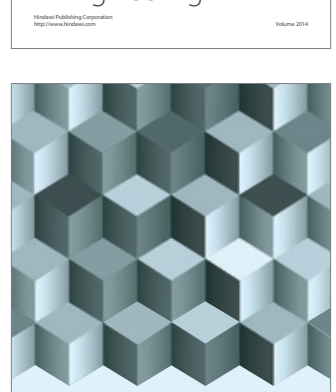

Journal of

Function Spaces
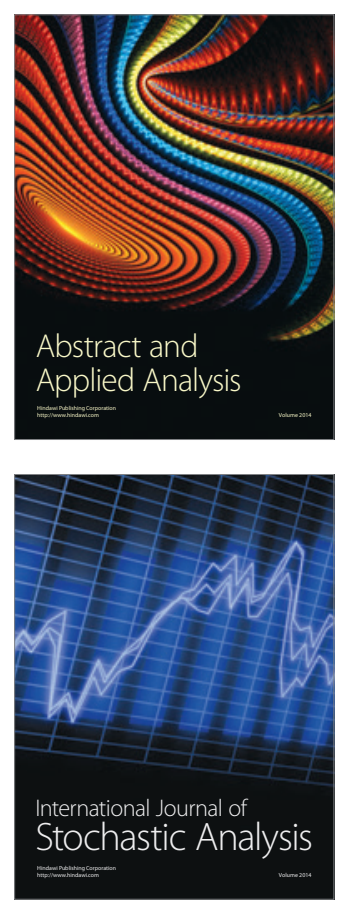

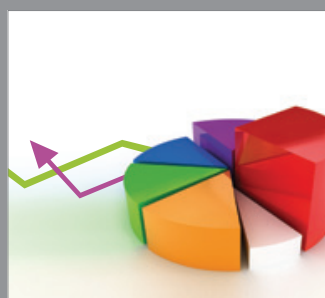

ournal of

Probability and Statistics

Promensencen
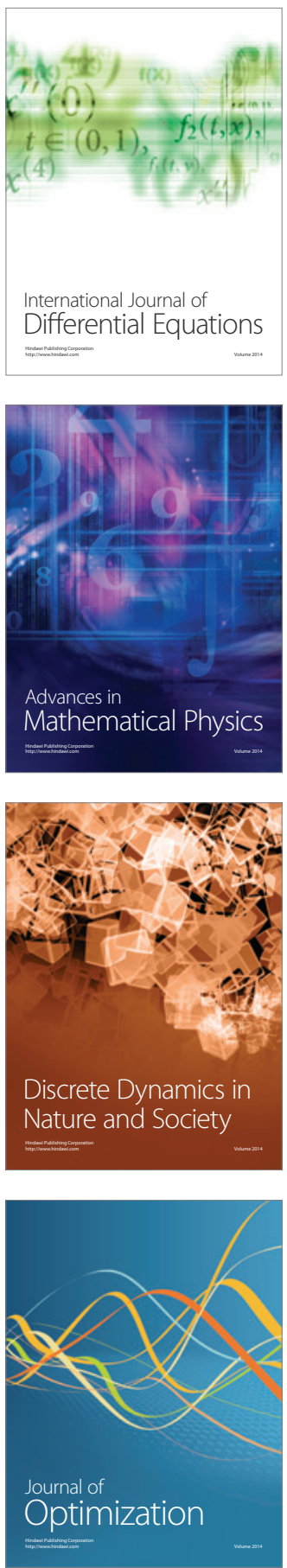